\title{
Rupture Spontanee Du Tendon D'achille Sur Terrain Diabetique : A Propos Du Cas
}

\author{
Ousseini Adakal (Chirurgie Générale) \\ Moussa Koini (Orthopédie-Traumatologie) \\ Université Dan Dicko Dankoulodo de Maradi, \\ Centre Hospitalier Régional de Maradi, Niger \\ Abdoulwahab Mohamed (Orthopédie-Traumatologie) \\ Université Abdou Moumouni de Niamey, \\ Hôpital National de Niamey, Niger \\ Sanoussi Akambi Kassoumou (Orthopédie-Traumatologie) \\ Harissou Adamou (Chirurgie Générale) \\ Université de Zinder, Hôpital National de Zinder, Niger \\ Ibrahim Amadou Magagi (Chirurgie Générale) \\ Hôpital National de Zinder (HNZ), Niger \\ Badjio Souna (Orthopédie-Traumatologie) \\ Université Abdou Moumouni de Niamey, \\ Hôpital National de Lamorde-Niamey, Niger
}

Doi: 10.19044/esj.2018.v14n6p295 URL:http://dx.doi.org/10.19044/esj.2018.v14n6p295

\begin{abstract}
Achilles Tendon Rupture (ATR) is a frequently described lesion in sports traumatology. It can occur following certain chronic or systemic diseases. We reported a case of a 48-year-old woman, a teacher by profession, who was consulting for lameness. Anamnesis found during a walk, a brutal sensation of blow on the heel with cowardice and sharp pain at the elective point imposing upon the resting of the patient. Clinical examination noted an increased spontaneous dorsiflexion of the affected ankle, depression corresponding to the tendon continuity solution, and a positive Thompson test. The diagnosis of Achilles Tendon Rupture was made. The patient was operated 2 weeks later by open surgery. A simple suture kept by a plaster splint was performed (the ankle in equinus). Functional rehabilitation was done for 6 weeks, and walking was allowed at the 6th week. In the absence of minimally invasive surgery, conventional surgery can provide good results in case of spontaneous Achilles Tendon Rupture at the cost of rigorous monitoring of the patient.
\end{abstract}


Keywords: Achilles tendon, Thompson's test, rupture of the tendon

\section{Résumé}

La rupture du tendon d'Achille (RTA) est une lésion fréquente en pathologie du sport. Elle peut survenir suite à certaines maladies chroniques ou systémiques. Nous rapportons le cas d'une femme âgée de 48 ans, enseignante, qui consultait pour boiterie. L'interrogatoire retrouvait à l'esquisse d'une marche, une sensation brutale de coup sur le talon avec lâchage et douleur vive au point électif imposant la mise au repos de la patiente. L'examen clinique montrait une dorsiflexion spontanée augmentée de la cheville atteinte, une dépression correspondant à la solution de continuité du tendon et un test de Thompson positif. Le diagnostic de rupture de tendon d'Achille était posé. La patiente était opérée 2 semaines après par une chirurgie à ciel ouvert. Une suture simple maintenue d'une attelle plâtrée a été réalisée, la cheville en équin. Une rééducation fonctionnelle était faite pendant 6 semaines. La marche était autorisée à la $6^{\text {ème }}$ semaine. À défaut de la chirurgie mini-invasive, la chirurgie classique peut donner des résultats satisfaisants en cas de RSTA au prix d'un suivi rigoureux du patient.

Mots clés : Tendon d'Achille, Test de Thompson, rupture du tendon.

\section{Introduction}

La rupture du tendon d'Achille (RTA) est une lésion fréquente en pathologie du sport (Leppilahti, 1998; Maffulli, 1998 ; Järvinen, 2005 ; Zakaria, 2014; Aktas, 2007). Elle peut survenir dans un contexte non traumatique, notamment suite à certaines maladies telles que le lupus érythémateux disséminé, la polyarthrite rhumatoïde, l'insuffisance rénale chronique, l'hyperparathyoïdie secondaire, le diabète et la prise de corticoïdes ou de fluoroquinolones (Zakaria, 2014; Maffulli, 2015; Benjilali, 2012; Assal, 2007 ; Newnham, 1991; Tezeren, 2006; Spoendlin, 2016). Son incidence augmente à travers le monde et est estimée à 18 pour 100.000 personnes (Leppilahti, 1998).

La forme aiguë spontanée est rare pouvant survenir sans cause apparente et le mécanisme est non élucidé (Benjilali, 2012 ; Assal, 2007). Si le diagnostic de la lésion est facile, le traitement reste encore controversé (Maffulli, 2011). Nous rapportons un cas de rupture spontanée du Tendon d'Achille (RSTA) chez une patiente diabétique et sous corticothérapie au long cours. 


\section{Observation}

Une patiente de 49 ans, enseignante de profession, consultait pour trouble de la marche liée à une douleur au niveau de sa cheville droite, de survenue brutale et ce sans contexte traumatique.

À l'anamnèse, le début de la symptomatologie remonterait à sept mois par des douleurs de la face postérieure de la cheville avec boiterie. Elle a été mise sous traitement médical à base d'antalgiques et anti-inflammatoires. L'évolution était marquée par la survenue brutale au cours de la marche d'une sensation de coup sur le talon avec lâchage et douleur l'obligeant à l'arrêt de la marche. Quelques instants après, elle reprenait la marche cependant, avec une incapacité de dérouler le pas (marche en trainant le pied). Comme antécédents, la patiente est asthmatique sous corticothérapie depuis 15 ans. Un diabète sous-jacent était découvert fortuitement à l'occasion du bilan pré opératoire (glycémie à $2.69 \mathrm{~g} / \mathrm{l}$.).

À l'examen physique, en décubitus ventral, genoux fléchis, on note une dorsiflexion spontanée augmentée de la cheville atteinte (Figure 1), une dépression correspondant à la solution de continuité du tendon et un test positif de Thompson. Face à ces signes, le diagnostic de rupture du tendon d'Achille était retenu. L'examen de la cheville controlatérale note une douleur à la palpation du talon. Ailleurs, l'examen des autres segments de membres et appareils reste sans particularité. Les résultats du bilan sanguin révélaient un taux de glycémie de $2.69 \mathrm{~g} / \mathrm{l}$.

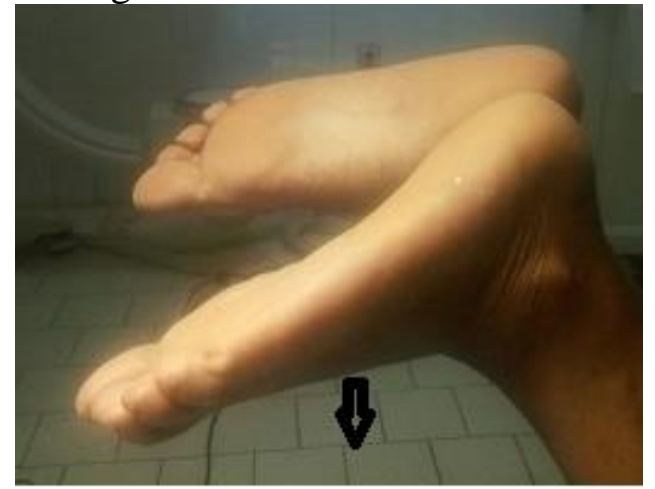

Figure 1. Dorsiflexion spontanée augmentée de la cheville.

Le traitement chirurgical à ciel ouvert était indiqué après l'équilibre diabétique obtenu sous régime et Metformine (Glucophage) ® cp 500mg à la posologie d'1 comprimé matin et soir. Sous rachi-anesthésie, patiente en décubitus ventral, garrot à la racine de la cuisse, billot sous la cheville, après préparation de tout le membre pelvien droit, nous avons procédé à un abord para achilléen médial, ouverture de la gaine aponévrotique, découverte d'un tendon très inflammatoire et boudiné dont la rupture est située à $4 \mathrm{~cm}$ de l'insertion calcanéenne (Figure 2A). 

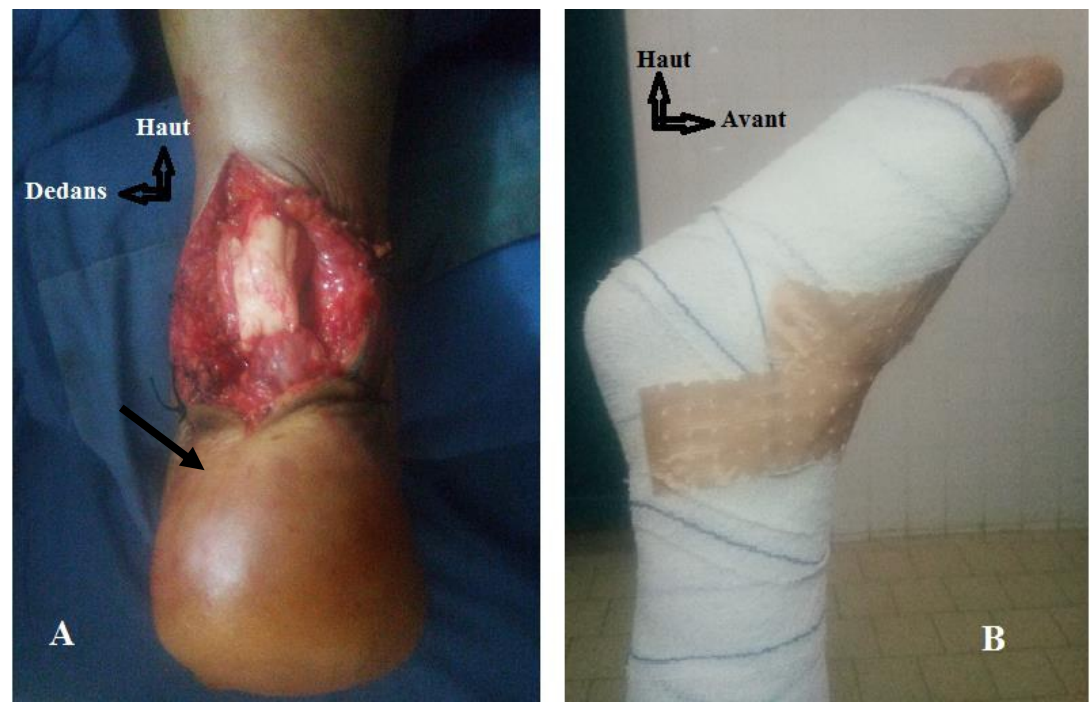

Figure 2. Images per opératoires. A : Tendon sectionné, inflammatoire et boudiné (flèche noire). B : Attelle suro-pédieuse postérieure en équin modéré du pied après suture tendineuse.

Après régularisation des deux extrémités, le pied en équin, on a réalisé une suture simple du tendon bout à bout par des points en $U$ à l'aide du Vicryl® 1 à résorption lente. Fermeture de la gaine tendineuse, du plan souscutané puis de la peau par des points séparés. Réalisation d'une attelle suropédieuse postérieure en équin modéré du pied (Figure2B) qui a été transformée en botte plâtrée après résorption des phénomènes œémateux inflammatoires pour une durée de 3 semaines. Une autre botte est confectionnée à 90 degrés du pied pour 3 autres semaines avec appui partiel. La rééducation fonctionnelle a débuté à la fin de la sixième semaine selon un rythme de 3 séances par semaine pendant 4 semaines. Le résultat à 6 mois de recul était satisfaisant tant sur le plan fonctionnel que sur le plan esthétique.

\section{Discussion}

Les ruptures aiguës du tendon d'Achille surviennent fréquemment lors des activités sportives de haut niveau ou de loisirs (Aktas, 2007 ; Maffulli, 2015 ; Assal, 2007). Le nombre et l'incidence de ces lésions tendineuses, en général, ont augmenté sensiblement au cours des dernières décennies. On estime qu'elles représentent $30 \%$ à $50 \%$ de toutes les lésions liées au sport (Järvinen, 2005). Leppilahti a estimé cette incidence dans la population générale à 8 pour 100.000 cas (Leppilahti, 1998). Ces lésions peuvent également s'observer sur terrain pathologique sous-jacent et constituer une entité particulière de ruptures dégénératives [Maffulli, 2015 ; Benjilali, 2012 ; Assal, 2007 ; Assal, 2007 ; Maffulli, 2011 ; Longo, 2009 ; Colné, 2011). Dans 
le cas de notre patiente, la rupture est survenue sur terrain diabétique de découverte inaugurale (glycémie à $2.69 \mathrm{~g} / \mathrm{l}$ ).

Les maladies hormonales, systémiques et métaboliques telles que le diabète sucré, le lupus érythémateux disséminé, l'hypercholestérolémie et l'obésité, le rhumatisme, la goutte semblent jouer un rôle primordial dans l'étiopathogénie des ruptures tendineuses (Maffulli 1999 ; Järvinen, 2005 ; Maffulli 2015 ; Newnham 1991 ; Longo 2009 ; Dakin 2017). Deux théories principales sont décrites : la «théorie dégénérative » et la «théorie mécanique ». Selon la théorie dégénérative, la dégénérescence chronique du tendon conduit à une rupture sans que des charges excessives ne soient appliquées. Cette dégénérescence peut résulter de plusieurs facteurs, y compris des altérations physiologiques du tendon, une surcharge chronique avec des microtraumatismes, un traitement pharmacologique et en association avec d'autres maladies (Longo, 2009). Certains médicaments tels que les corticoïdes, les fluoroquinolones présentent aussi le risque de rupture tendineuse lorsqu'ils sont administrés sur une longue période (Maffulli, 2011 ; Longo, 2009).

Dans notre observation, la rupture s'est produite chez une femme âgée de 48 ans avec des tares préexistantes : un diabète de découverte inaugurale, un asthme évoluant depuis une quinzaine d'années et un antécédent de douleurs achilléennes chroniques prises en charge par des anti-inflammatoires non stéroïdiens. L'asthme évolue par crises épisodiques et la prise en charge a toujours fait appel, en plus des bronchodilatateurs, aux corticoïdes. Une relation de cause à effet a été rapportée entre l'utilisation à longue durée de corticostéroïdes et la RSTA (Benjilali, 2012 ; Newnham, 1991). Il est difficile d'établir la part de chaque facteur dans la survenue de la rupture, mais l'association multifactorielle peut majorer le risque (Maffulli, 2011). Pour ce qui est du diagnostic facile, le tableau clinique d'une RTA est typique chez la plupart des patients et l'anamnèse suffit à poser le diagnostic. Le patient rapporte l'apparition soudaine d'un lâchage brusque accompagné d'une douleur élective au niveau de la région postérieure de la cheville. Il a souvent le sentiment « d'avoir été frappé par l'arrière » (Benjilali, 2012 ; Assal, 2007 ; Mahdane, 2014).

Le diagnostic dans le cas de notre observation était posé essentiellement sur la base de l'anamnèse (sensation brutale au cours de la marche du coup sur le talon avec lâchage et douleurs l'obligeant à l'arrêt de la marche) et de l'examen physique (une dorsiflexion spontanée augmentée de la cheville atteinte, une dépression correspondant à la solution de continuité $\mathrm{du}$ tendon et un test positif de Thompson. Cependant, le diagnostic peut être difficile dans certains cas. Environ, 20\% des ruptures aiguës ne sont pas diagnostiquées lors de la première consultation (Assal 2007). Dans ce cas, 
l'échographie est un bon moyen diagnostique précisément dans les cas de rupture partielle (Assal, 2007 ; Maffulli, 2011).

L'évaluation d'une lésion du tendon d'Achille par l'IRM garde tout son intérêt dans le suivi de la cicatrisation au cours du traitement non-opératoire ou en cas de doute diagnostique de rupture partielle ou itérative. (Weber, 2003). Plusieurs procédés thérapeutiques ont été décrits pour la prise en charge des RTA (Aktas, 2007 ; Assal, 2007 ; Colné, 2011 ; Mahdane, 2014 ; Weber, 2003 ; Rebeccato, 2001).

La pluralité de techniques dénote le manque d'approche thérapeutique consensuelle dans le traitement des ruptures du tendon d'Achille. Habituellement, deux types de traitements sont utilisés à savoir, le traitement orthopédique ou chirurgical. Le traitement chirurgical est indiqué surtout chez les sportifs de haut niveau ou les adultes en pleine activité physique (Järvinen, 2005 ; Maffulli 2015 ; Longo, 2009 ; Aktas, 2007 ; Rebeccato, 2001). Il présente l'avantage d'une cicatrisation plus rapide, plus solide et un risque de rerupture moindre (Aktas, 2007 ; Weber 2003). Le traitement non chirurgical doit être réservé aux patients sédentaires, d'un âge avancé ou présentant des maladies systémiques nécessitant une corticothérapie au long cours. (Assal, 2007 ; Ramirez, 2007). Selon Weber, il n'y a pas de différence significative qu'il s'agisse du traitement orthopédique ou sanglant (Weber, 2003). Dans le cas ci-rapporté, nous avons utilisé la chirurgie à ciel ouvert avec une suture simple du tendon dite de «bout en bout ». Le choix du traitement chirurgical chez notre patiente était guidé par un souci d'une réhabilitation précoce (enseignante de profession). La chirurgie mini-invasive est une technique simple qui peut être réalisée en ambulatoire et nécessite un minimum de soins de suivi. Elle est indiquée en cas d'échec du traitement conservateur ou en première intention permettant ainsi de diminuer les infections et les nécroses cutanées liées à la chirurgie classique (Maffulli, 2001; Longo 2009; Mahdane, 2014 ; Tam, 2017). Selon certains auteurs, la thromboprophylaxie à base d'héparine de bas poids moléculaire est instituée pour une durée de 6 semaines après l'intervention (Maffulli, 1999). Ramirez recommande cette prophylaxie thromboembolique surtout en cas de rupture bilatérale survenant sur terrain diabétique (Ramirez, 2007). Chez notre patiente et pour d'autres auteurs (Newnham, 1991; Longo 2009), cette prophylaxie n'est pas systématique. Le protocole de rééducation le plus communément utilisé est l'immobilisation avec un plâtre suro-pédieux en équin pendant 4 semaines, le pied en position plus neutre pendant 4 semaines supplémentaires (Maffulli, 2015 ; Longo, 2009). Quelle que soit la technique utilisée, l'immobilisation pour le traitement des ruptures du tendon calcanéen est obligatoire et se fait en deux étapes. Dans un premier temps, la cheville est immobilisée en équin dans un plâtre ou une orthèse pour 3 semaines puis dans un second temps, un autre plâtre est confectionné, la cheville à 90 degrés par rapport à l'axe de la jambe 
pour 3 semaines suplémentaires (Aktas, 2007). Notre patiente a été immobilisée par plâtre pour une durée de 6 semaines au total. La rééducation est un complément indispensable. Plusieurs types d'exercices sont proposés aux patients : dorsiflexion et flexion plantaire pour récupérer l'amplitude des mouvements articulaires, renforcement de la force du triceps : physiothérapie, natation, escaliers (Aktas, 2007 ; Benjilali, 2012). Dans notre observation, la patiente a bénéficié de 3 séances de kinésithérapie par semaine pendant 4 semaines visant à récupérer les différents mouvements de la cheville. Malgré une chirurgie à ciel ouvert sur terrain à risque, notre résultat immédiat (recul de 6 mois) est satisfaisant sur le plan fonctionnel et esthétique.

\section{Conclusion}

La rupture spontanée du tendon d'Achille doit faire rechercher une tare dégénérative préexistante ou une corticothérapie au long cours. À défaut de la chirurgie mini-invasive, la chirurgie à ciel ouvert donne des résultats satisfaisants en cas de RSTA au prix d'un suivi rigoureux du patient.

\section{References:}

1. Aktas, S., Kocaoglu, B., Nalbantoglu, U., Seyhan, M. \& Guven, O. (2007). End-to-End Versus Augmented Repair in the Treatment of Acute Achilles Tendon Ruptures. J Foot Ankle Surg. 46 (5):336-40.

2. Assal, M. (2007). Rupture aiguë du tendon d'Achille. Actualité diagnostique et thérapeutique. Schweiz $\mathrm{Z}$ Fü Sportmed Sporttraumatologie. 55 (1):5-10.

3. Benjilali, L., Benhima, H., Zahlane, M. \& Essaadouni, L. (2012). Rupture spontanée du tendon d'Achille à la phase précoce d'un lupus érythémateux systémique. Rev Médecine Interne. 33 (8) :e47-8.

4. Colné, P. (2011). Rupture du tendon d'Achille et réparation percutanée. Kinésithérapie Rev. 11(113):10.

5. Dakin, SG., Newton, J., Martinez, FO., Hedley, R., Gwilym, S., \& Jones, N. (2017). Chronic inflammation is a feature of Achilles tendinopathy and rupture. Br J Sports Med. 098161.

6. Järvinen, TA., Kannus, P., Maffulli, N. \& Khan, KM. (2005). Achilles tendon disorders: etiology and epidemiology. Foot Ankle Clin. 10 (2):255-266.

7. Leppilahti, J. \& Orava, S. (1998). Total Achilles tendon rupture. Sports Med.; 25 (2):79-100.

8. Longo, UG., Ronga, M. \& Maffulli, N. (2009). Acute ruptures of the achilles tendon. Sports Med Arthrosc Rev. 17(2):127-138.

9. Maffulli, N. (1999). Current Concepts Review-Rupture of the Achilles Tendon. JBJS. 81 (7):1019-36. 
10. Maffulli, N., Longo, UG., Loppini, M. \& Denaro, V. (2010). Current treatment options for tendinopathy. Expert Opin Pharmacother. 11(13):2177-86.

11. Maffulli, N., Longo, UG., Maffulli, GD., Khanna, A. \& Denaro, V. (2011). Achilles tendon ruptures in diabetic patients. Arch Orthop Trauma Surg. 131 (1):33-8.

12. Maffulli, N., Via, AG. \& Oliva, F. (2015). Chronic Achilles Tendon Disorders. Clin Sports Med. 34(4):607-24.

13. Mahdane, H., Khaissidi, A., Hammou, N., Elidrissi, M., Mohamed, S., \& Elibrahim, A. (2014). Traitement chirurgical à ciel ouvert des ruptures récentes du tendon calcanéen. Pan Afr Med J. 18. http://www.panafrican-med-journal.com/content/article/18/144/full/

14. Newnham, DM., Douglas, JG., Legge, JS. \& Friend, JA. (1991). Achilles tendon rupture: an underrated complication of corticosteroid treatment. Thorax. 46 (11):853-854.

15. Ramirez, MA. \& Richardson, LC. (2007). Pulmonary Embolism Associated with Spontaneous Bilateral Achilles Tendon Rupture. J Foot Ankle Surg. 46 (4):283-7.

16. Rebeccato, A., Santini, S., Salmaso, G. \& Nogarin, L. (2001). Repair of the Achilles tendon rupture: a functional comparison of three surgical techniques. J Foot Ankle Surg. 40 (4):188-194.

17. Spoendlin, J., Meier, C., Jick, SS. \& Meier, CR. (2016). Achilles or biceps tendon rupture in women and men with type 2 diabetes: A population-based case-control study. J Diabetes Complications. 30 (5):903-9.

18. Tam, KF. \& Lui, TH. (2017). MR Imaging of the Achilles Tendon after Surgical Repair. Open Orthop J. 11 (Suppl-4, M10):697-703.

19. Tezeren, G. \& Kuru, I. (2006). Augmentation vs nonaugmentation techniques for open repairs of Achilles tendon ruptures with early functional treatment: a prospective randomized study. J Sports Sci Med. 5 (4):607.

20. Weber, M., Niemann, M., Lanz, R. \& Müller, T. (2003). Nonoperative treatment of acute rupture of the Achilles tendon. Am J Sports Med. 31 (5):685-691.

21. Zakaria, MHB., Davis, WA. \& Davis, TME. (2014). Incidence and predictors of hospitalization for tendon rupture in Type 2 diabetes: the Fremantle Diabetes Study. Diabet Med. 31 (4):425-30. 